\title{
A clinical study to evaluate incidence of dry eye following cataract surgery
}

\author{
Abhinav Khadke ${ }^{1}$, Mansur Ali Khan ${ }^{2, *}$, P S Moulick ${ }^{3}$, Sandeep Gupta ${ }^{4}$, Sandeep Shankar ${ }^{5}$ \\ ${ }^{1}$ Resident, Dept. of Ophthalmology, Armed Forces Medical College, Pune, ${ }^{2}$ Professor and HOD, Dept. of Ophthalmology, \\ Command Hospital Air Force, Bangalore, ${ }^{3}$ Professor and HOD, Dept. of Ophthalmology, Armed Forces Medical College, Pune, \\ Maharashtra, ${ }^{4}$ Associate Professor, Dept. of Ophthalmology, Command Hospital, Kolkata, West Bengal, ${ }^{5}$ Associate Professor, \\ Dept. of Ophthalmology, Armed Forces Medical College, Pune, Maharashtra, India
}

*Corresponding Author:

Email: mansurophthal@gmail.com

\begin{abstract}
Introduction: Post cataract surgery dry eye in the susceptible population can adversely impact visual outcome and overall patient comfort. There are varying reports on the incidence of post cataract surgery dry eye depending on patient population and surgical technique. This study has evaluated the incidence and factors causing dry eye following phacoemulsification, small incisional cataract surgery (SICS) and conventional extracapsular cataract surgery (ECCE).

Materials and Methods: Patients with cataract in the age group of 40 to 80 years with normal ocular surface and schirmers test at baseline were followed up after cataract surgery with schirmers recording at 1 month and 3 months. Pre and post- surgery schirmers values were compared for estimation of dry eyes.

Results: A total 390 patients underwent cataract surgery with an overall post surgery residual dry eye incidence of $22.1 \%(95 \%$ CI 17.94 to 26.17 ) at the end of 3 months follow up. Of these $31 \%$ had mild, $67 \%$ had moderate and $2 \%$ had severe dry eye. A higher incidence was seen in SICS as compared to phacoemulsification. A statistically significant association was noted between dry eye and diminution of vision $(\mathrm{p}=0.001)$.
\end{abstract}

Keywords: Dry eye, Extra capsular, Phacoemulsification, Small incision, Schirmers test.

\section{Introduction}

Excellent visual outcomes that is usually expected after cataract surgery can sometimes be affected by occurrence of dry eye as has been reported by several studies. ${ }^{1-3}$ Dry eye is a multifactorial disease of the tear film and the ocular surface, that results in symptoms of discomfort, visual disturbance, and tear film instability, with potential damage to the ocular surface as reported. ${ }^{4-6}$ The dry eye occurring after cataract surgery is hypothesized to be due to the changes in the ocular surface due to the surgical trauma and post operative inflammation. ${ }^{7}$ However despite cataract surgery being so common, large studies on post cataract surgery dry eye are scarce and symptoms attributable to dry eye are not specifically documented. After cataract surgery many patients complain of foreign body sensation, irritation, redness and blurring of vision which are considered as unwanted effects of the surgery. ${ }^{8}$ These effects are worse in the elderly population and in some cases may persist and develop into a full fledged dry eye syndrome requiring treatment. Thus, inspite of a perfect cataract surgery and a good snellen's visual acuity the patients may remain dissatisfied. Occurrence of dry eye following cataract surgery can affect the functional recovery and therefore it is important to know the incidence of dry eye after cataract surgery and the factors associated with this type of dry eye. ${ }^{9}$ Most of the previous studies on this subject have estimated dry eye following small incisional cataract surgery but there are very few studies especially in India on dry eye occurring after phacoemulsification which is a major part of our study.

\section{Materials and Methods}

This prospective observational study was conducted at a tertiary care hospital between August 2014 and August 2016. The study was approved by institutional ethics committee. The study sample was drawn from patients reporting with visually significant cataract to the ophthalmology OPD. The sample size was calculated as 384 taking an estimated incidence of $66.2 \%$ with $95 \%$ confidence limits. All patients underwent a detailed ocular examination including slit lamp examination as a part of work up for cataract surgery and also to rule out any ocular surface disorder. Only patients in the age group of $40-80$ years finally listed for cataract surgery were included in the study. Those with pre-existing dry eyes (schirmers less than $10 \mathrm{~mm}$ ), Sjogren's syndrome, pre-existing ocular diseases like glaucoma, uveitis, disorders of lid and nasolacrimal pathway, ocular allergies, pterygium and previous ocular surgeries were excluded from the study. The study parameter for documenting dry eye was the Schirmer's strip wetting at the end of 5 min (basal and reflex). Schirmers test was done at baseline before surgery and at one month and 3 months after surgery in the operated eye. Schirmer test value less than $10 \mathrm{~mm}$ was taken as dry eye. Dry eye was graded as mild, moderate and severe based on Schirmer's test values 7$9 \mathrm{~mm}, 5-7 \mathrm{~mm}$ and $<5 \mathrm{~mm}$ respectively. In addition data was maintained separately for each individual regarding age, gender, pre operative and post operative vision and type of surgery. The results were analysed at one month and three months of follow up. Statistical evaluation was done with SPSS for IBM version 20. 
Chi square tests and fischers exact tests were used for testing statistical significance and $\mathrm{p}$ value less than 0.05 was taken as significant.

\section{Results}

The baseline characteristics of the sample population are listed in Table 1. Maximum patient population operated for cataract were in the age group of $61-70 \mathrm{yrs}(40.3 \%)$. Gender wise distribution showed that study included $53.3 \%$ males and $46.7 \%$ females operated for cataract surgery. Out of 390 patients 380 underwent phacoemulsification surgery, 9 underwent SICS while ECCE was done only for single patient. The cumulative incidence of dry eyes at the end of three months was $22.1 \%$ (86/390) Table 2 and Graph 1. Fifty six patients were found to have dry eyes at 1 month of which 28 resolved and 28 persisted at 3 months and 30 more new patients developed dry eyes at 3 months. Majority of patients had mild dry eyes. Group wise distribution of post cataract surgery dry eyes is listed in Table 3. There was no significant difference in the occurrence of dry eyes in older age group (>50 versus < 50 years, $\mathrm{p}=0.418)$. Similarly no significant difference was seen between males and females so far as incidence of post cataract surgery dry eyes (males vs females $p$ $=0.903$ ). More cases of dry eyes were seen after SICS as compared to phacoemulsification and the difference was statistically significant $(p=0.001)$. Analysis of visual recovery between patients who developed dry eyes after surgery versus those who did not have dry eyes, showed visual recovery significantly worse in those who developed dry eyes with vision below 6/12 more often seen in patients with dry eyes, Table 4 and Graph 2. However the very poor vision seen in 6 cases $(<6 / 24)$ was due to age related macular degeneration and not related to dry eye.

Table 1: Demographic data and study sample baseline characteristics

\begin{tabular}{|c|c|c|c|c|c|c|}
\hline \multicolumn{2}{|c|}{ Age Distribution: \% } & Gender Distribution \% & \multicolumn{2}{c|}{ Type of Surgery \% } \\
\hline$<50$ years & $>50$ years & Male & Female & Phacoemulsification & SICS & $\begin{array}{c}\text { Conventional } \\
\text { ECCE }\end{array}$ \\
\hline $15.12 \%$ & $84.9 \%$ & $53.3 \%$ & $46.7 \%$ & $97.4 \%$ & $2.3 \%$ & $0.3 \%$ \\
\hline
\end{tabular}

Table 2: Post Cataract surgery incidence and severity of dry eyes at 1 month and 3 months

\begin{tabular}{|l|c|c|c|}
\hline \multicolumn{2}{|l|}{$\mathbf{n}=\mathbf{3 9 0}$} & At 1 Month (\%) & At 3 Months (\%) \\
\hline \multicolumn{2}{|l|}{ Incidence of Dry eyes } & $14.35 \%$ & $14.87 \%$ \\
\hline \multirow{2}{*}{$\begin{array}{l}\text { Distribution } \\
\text { as per severity }\end{array}$} & Mild & $87.5 \%$ & $31 \%$ \\
\cline { 2 - 4 } & Moderate & $5.5 \%$ & $67 \%$ \\
\cline { 2 - 4 } & Severe & $7 \%$ & $2 \%$ \\
\hline
\end{tabular}

Table 3: Distribution of dry eyes post cataract surgery group wise at final follow up (3 months)

\begin{tabular}{|l|c|c|c|}
\hline $\mathbf{n = 3 9 0}$ & & $\mathbf{\%}$ & \multirow{2}{*}{ P value } \\
\hline \multirow{3}{*}{ Age } & $<50$ years & $20.9 \%$ & 0.418 \\
\cline { 2 - 3 } & $>50$ years & $79.1 \%$ & \\
\hline \multirow{3}{*}{ Gender } & Male & $52 \%$ & \multirow{2}{*}{0.903} \\
\cline { 2 - 3 } & Female & $48 \%$ & \\
\hline \multirow{2}{*}{ Type of Surgery } & Phacoemulsification & $97.4 \%$ & \multirow{2}{*}{0.001} \\
\cline { 2 - 3 } & SICS & $2.3 \%$ & \\
\cline { 2 - 3 } & Conventional ECCE & $0.3 \%$ & \\
\hline
\end{tabular}

Table 4: Post cataract surgery recovery of Vision: dry eye cases versus those without dry eyes

\begin{tabular}{|c|c|c|c|c|}
\hline $\mathbf{n}=390$ & Dry eye cases (\%) & Without dry Eye (\%) & P value \\
\hline \multirow{3}{*}{ Vision } & $6 / 9$ or above & $16.16 \%$ & $83.84 \%$ & \\
\cline { 2 - 4 } & $6 / 12$ & $28.99 \%$ & $71.01 \%$ & \multirow{3}{*}{0.001} \\
\cline { 2 - 4 } & $6 / 18$ & $52.94 \%$ & $47.06 \%$ & \\
\cline { 2 - 4 } & $6 / 24$ & $0.00 \%$ & $100.00 \%$ & \\
\cline { 2 - 5 } & $6 / 60$ & $0.00 \%$ & $100.00 \%$ & \\
\hline
\end{tabular}


Graph 1: Graph showing incidence of dry eyes at 1 month and at 3 month

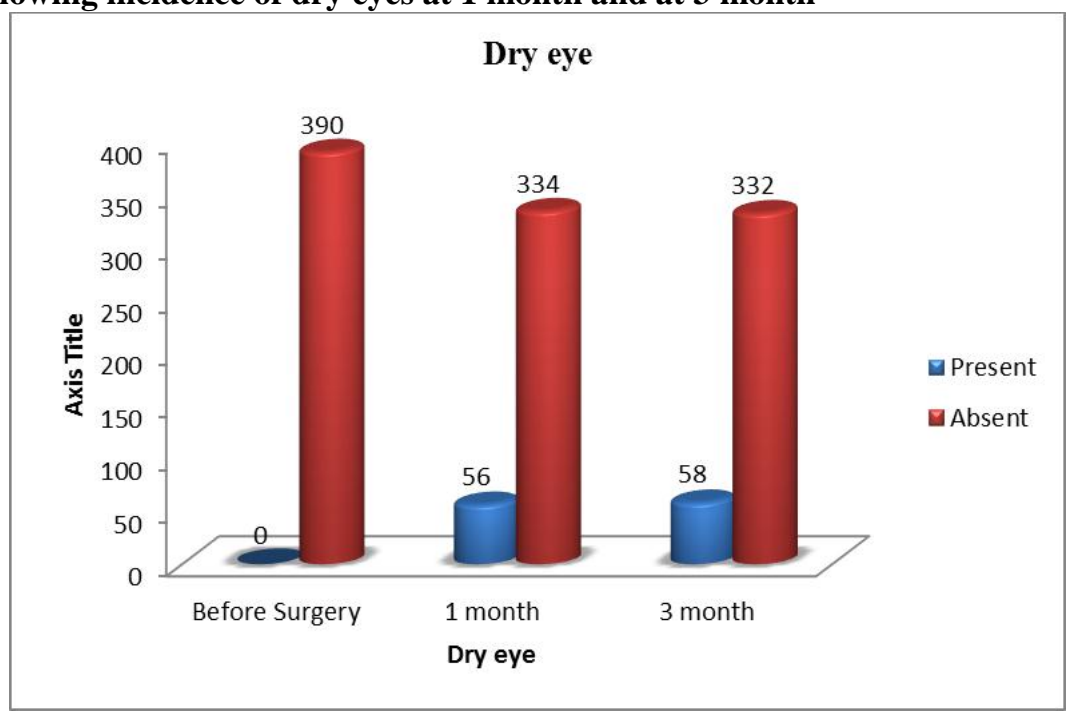

Graph 2: Graph showing correlation of visual acuity and dry eye

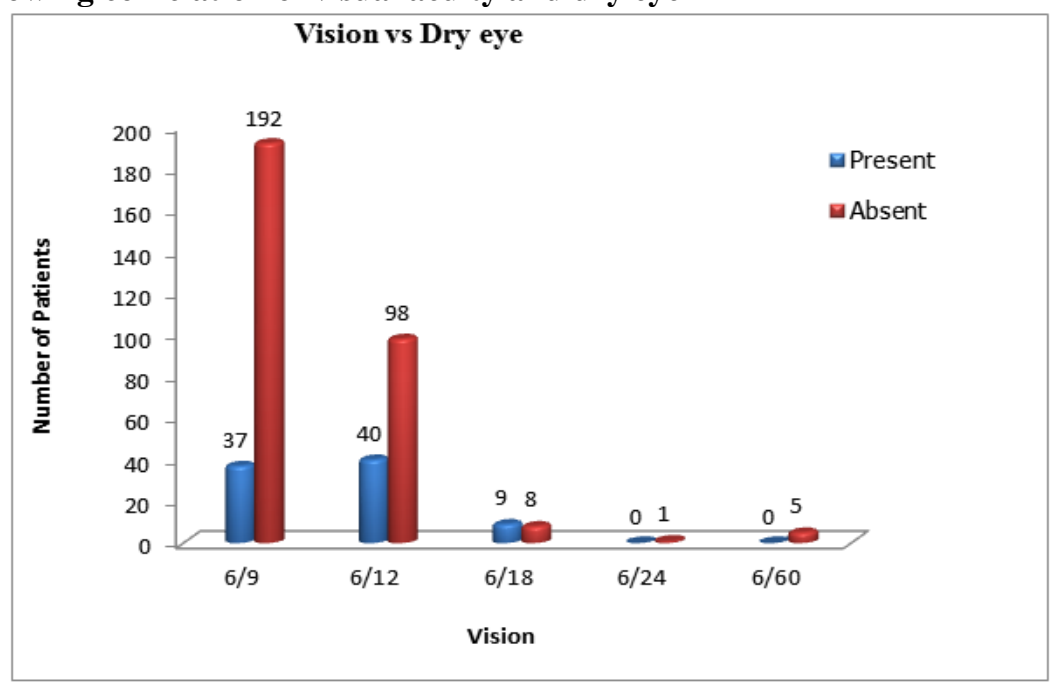

\section{Discussion}

This prospective study carried out to estimate the incidence of dry eyes after cataract surgery reiterates findings of previous studies and also brings out important variations in comparison to similar studies on post cataract surgery dry eyes. ${ }^{10-12}$ One important differentiating feature was that in this study most cases underwent phacoemulsification and only a few cases were of SICS and ECCE. We have also compared the dry eye incidence between phacoemulsification and SICS. On extensive literature search only one study by Sinha et al is found in which SICS and phacoemulsification were compared for development of dry eye post surgery which were $43.5 \%$ and $56.5 \%$ respectively. ${ }^{10}$ In our study the overall incidence (22.1\%) was lower and there was a significant difference between Phaco and SICS with SICS showing a much higher incidence. Visual acuity for each patient at post-operative 3 month with snellen's chart showed that maximum patients had 6/9 vision (58.7\%), followed by $6 / 12$ vision in 138 patients (35.4\%). Intermittent blurring of vision as the tear film breaks in between the blinks has been reported in cases of post cataract surgery dry eyes but in our study we found there is diminution of vision or failure to gain complete visual recovery post cataract surgery in cases of dry eyes.

The reported Incidence of dry eye after cataract surgery varies from $9.8 \%$ to $66.2 \%$ depending upon whether it was predominantly phacoemulsification or SICS. Kasetsuwan Ngamjit, et al showed that the incidence of dry eye in patients who all underwent phacoemusification was $9.8 \% .{ }^{11}$ Kavitha et al found that after manual small incision cataract surgeries with corneoscleral tunnel incisions, $66.2 \%$ of the patients had dry eyes, which was relatively high. ${ }^{12}$ The $22.1 \%$ incidence in our study is in between the above quoted studies as our sample consisted of both SICS, 
phacoemulsification and conventional ECCE of which phacoemulsification constituted the majority. The difference in incidence could also be due to the criteria used for diagnosis of dry eyes. In a study using impression cytology for detecting dry eyes in cases who underwent either SICS or phacoemulsification, the incidence of dry eyes was similar to our study though we have used schirmer's test to diagnose dry eyes. ${ }^{10}$ In our study age did not have a significant association with incidence of dry eyes after cataract surgery. Kamla Dodia et al reported post phacoemulsification cataract surgery showed association of dry eye with higher age (>65 yrs). ${ }^{13}$ Cataract and dry eye eyes both are age related and the association in that study could be just incidental rather than causal. We did not find any significant difference for the occurrence of dry eyes post cataract surgery between the two genders. This contrasts with other studies which have reported a higher prevalence of the dry eye in females than in males in the general population. ${ }^{14,15}$ An association of dry eyes with postmenopausal females is well known. The results in our study may have been influenced by a larger number of males undergoing SICS as compared to females. A stronger association of SICS with development of dry eye is in consonance with study by Kavitha et al who showed a strong association of SICS having with development of dry eye. ${ }^{12}$ This may be due to shorter duration of phacoemulsification, shorter microscope light exposure and the faster visual rehabilitation permitting rapid tapering of topical medications. A larger incision in SICS leads to more corneal denervation resulting in tear film abnormality and dry eye.

To conclude dry eye is known to have various etiological factors out of which cataract surgery is one of the factors predisposing to dry eye. This study showed $22.1 \%$ cumulative incidence of dry eye in patients operated for cataract. In this study only schirmer tear strip was used to diagnose dry eye which is a common OPD test to diagnose dry eyes. In our study phacoemusification was the predominant surgery done in the study sample so the incidence in our study mainly reflects dry eye after phacoemulsification but there was a statistically significant association of SICS with postoperative dry eye as compared to phacoemulsification. Eyes with post operative dry eye may also have lesser visual recovery compared to those who do not develop significant dryness.

\section{References}

1. Zabel RW, Mintsioulis G, MacDonald IM, Valberg J, Tuft SJ. Corneal toxic changes after cataract extraction. Can J Ophthalmol. 1989 Dec;24(7):311-6.

2. Srinivasan R, Agarwal V, Suchismitha T, Kavitha S. Dry eye after Phacoemulsification. AIOC Proceedings. 2008.

3. Roberts CW. Dry eye symptoms following cataract surgery. Invest Ophthalmol Vis Sci. 2006 May 1;47(13):232.
4. Lemp MA, Baudouin C, Baum J et al. The definition and classification of dry eye disease: report of the Definition and Classification Subcommittee of the International Dry Eye Work Shop (2007). Ocul Surf. 2007 Apr 1;5(2):7592.

5. Sahai A, Malik P. Dry eye: prevalence and attributable risk factors in a hospital-based population. Indian $J$ Ophthalmol. 2005 Apr 1;53(2):87.

6. Fernando Murrilo LS, MJMEJH JH. Dry eye. Cornea.1997:663-86.

7. Li XM, Hu L, Hu J, Wang W. Investigation of dry eye disease and analysis of the pathogenic factors in patients after cataract surgery. Cornea 2007 Oct 1;26:S16-20.

8. Cho YK, Kim MS. Dry eye after cataract surgery and associated intraoperative risk factors. Korean $J$ Ophthalmol. 2009 Jun 1;23(2):65-73.

9. Nistor MC, Nistor C. Clinical correlations between dry eye and cataract surgery. Oftalmologia. 2006 Dec;51(4):79-82.

10. Sinha M, Sinha A, Chowdhury B. Comparative Evaluation of Dry Eye Following Cataract Surgery: A Study from North India. IOSR Journal of Dental and Medical Sciences. 2014;13(6):13-8.

11. Kasetsuwan N, Satitpitakul V, Changul T, Jariyakosol S. Incidence and pattern of dry eye after cataract surgery. PloS one. 2013 Nov 12;8(11):e78657.

12. Venugopal KC, Krishnaraj PA, Chandan N. Evaluation of Dryness of Eyes after Manual Small Incision Cataract Surgery with Corneoscleral Tunnel Incision. J Clin Diagn Res. 2012 Aug 1;6(6):1029-33.

13. Dodia K, Bapat S, Chudasama RK. Dry eye risk factors after phacoemulsification cataract surgery at a secondary care hospital. Int J Health Allied Sci. 2013 Oct $1 ; 2(4): 242$.

14. Hikichi T, Yoshida A, Fukui Y et al. Prevalence of dry eye in Japanese eye centers. Graefe's Arch clin Exp Ophthalmol. 1995 Sep 1;233(9):555-8.

15. Cetinkaya S, Mestan E, Acir NO, Cetinkaya YF, Dadaci $\mathrm{Z}$, Yener HI. The course of dry eye after phacoemulsification surgery. BMC ophthalmol. 2015 Jun 30;15(1):68. 